Document downloaded from:

http://hdl.handle.net/10251/49226

This paper must be cited as:

Verdú Martín, GJ.; Ginestar Peiro, D. (2014). Modal decomposition method for BWR stability analysis using Alpha-modes. Annals of Nuclear Energy. 67:31-40. doi:10.1016/j.anucene.2013.07.035.

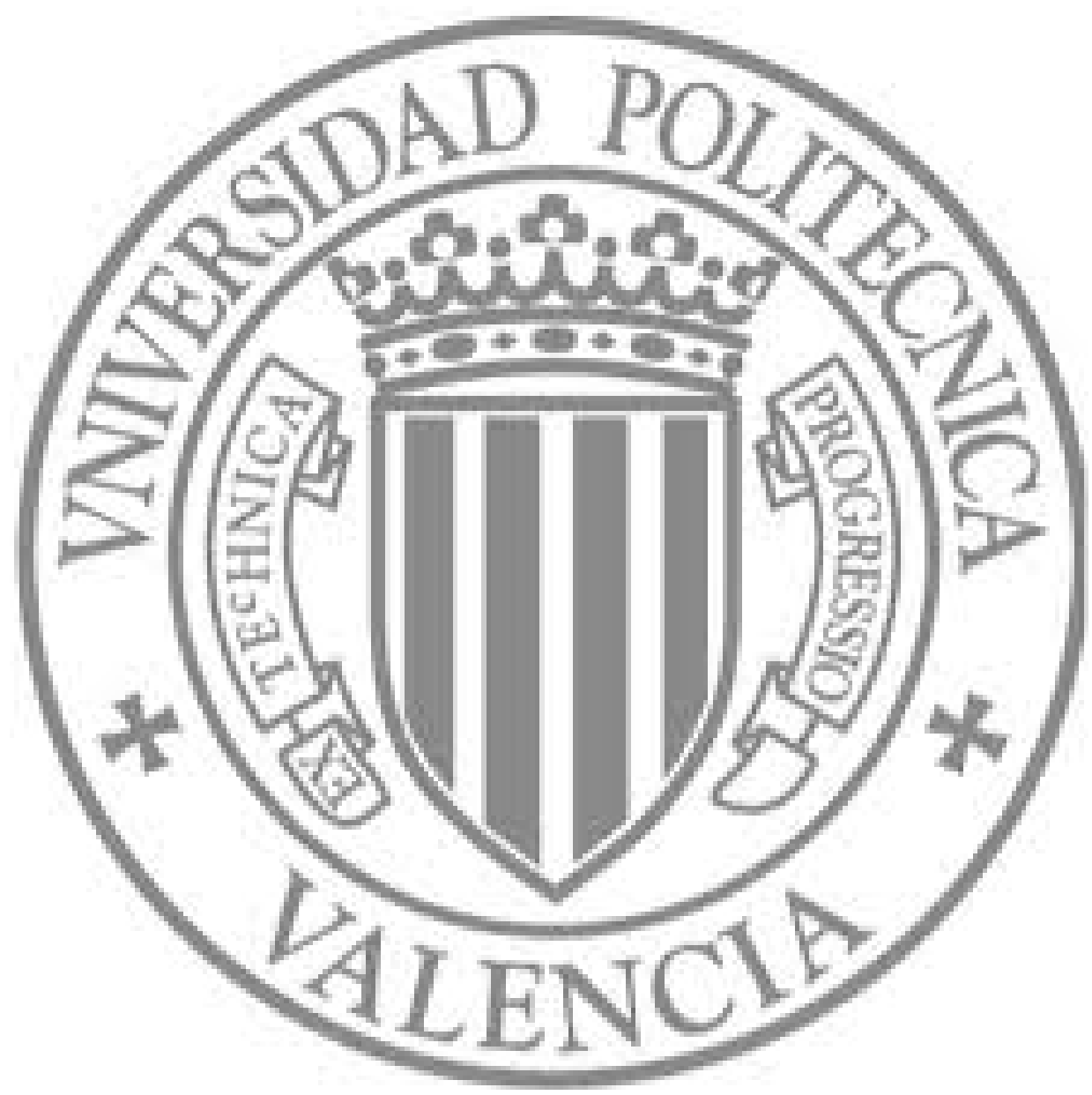

The final publication is available at

http://dx.doi.org/10.1016/j.anucene.2013.07.035

Copyright Elsevier Masson 


\title{
Modal Decomposition Method for BWR Stability Analysis using Alpha-Modes
}

\author{
G. Verdú ${ }^{\mathrm{a}}$ D. Ginestar ${ }^{\mathrm{b}, *}$

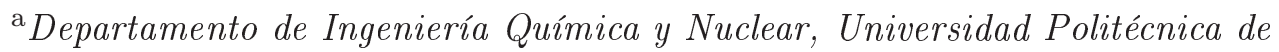 \\ Valencia. Camino de Vera, 14. Valencia. Spain. \\ ${ }^{\mathrm{b}}$ Instituto de Matemática Multidisciplinar, Universidad Politécnica de Valencia. \\ Camino de Vera, 14. Valencia. Spain.
}

\begin{abstract}
In-phase and out-of-phase oscillations have been observed in BWR reactors. To improve the safety of these reactors it is necessary to detect in a reliable way these oscillations from the neutronic signals. In this paper, a methodology to decompose the neutronic signals in its modal amplitudes is proposed. Usually, to compute this decomposition the Lambda eigenfunctions are used as expansion functions and their adjoint modes are used as weight factors. Different approaches using the Alpha modes are investigated to obtain the LPRM signals modal decomposition for a BWR unstability event. The calculation of Alpha eigenmodes is reviewd and the oscillation parameters for the modal decomposition of the neutronic signals from Ringhals NPP have been calculated.
\end{abstract}

Key words: Lambda Modes, Alpha Modes, BWR reactors stability, LPRM readings.

\section{Introduction}

Several events have been observed in BWR nuclear power reactors where fully developed oscillations were present in the neutronic power measured by the LPRM detectors installed in the reactor core. Some of these events were involuntary and other ones were induced intentionally as experiments. Mainly, BWR neutronic oscillations have been classified as in-phase oscillations, where

\footnotetext{
* Corresponding author.

Email addresses: gverdu@iqn.upv.es (G. Verdú), dginesta@mat.upv.es (D. Ginestar ).
} 


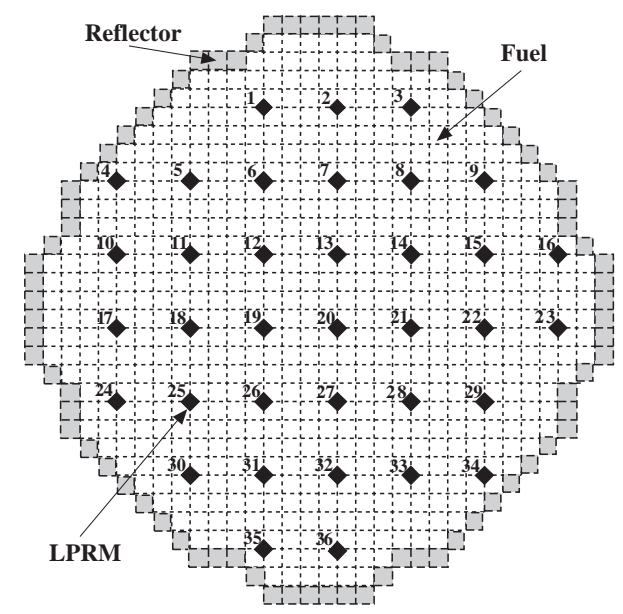

Fig. 1. LPRMs disposition in an axial plane for Ringhals 1 reactor.

the whole core oscillates together. And the out-of-phase oscillations, where half of the core behaves out-of-phase from the other half. A modal interpretation has given for the in-phase and the out-of-phase oscillations of a BWR reactor, associating the in-phase oscillations with the amplitude of the fundamental mode of the reactor. The out-of-phase oscillations are interpreted as the contribution of the subcritical azimuthal modes amplitudes to the oscillations (March-Leuba and Rey, 1993).

For safe operation, it is desirable to have a monitoring system capable of indicating the change in neutron multiplication within the reactor core as criticality is approached. The neutron monitoring system in a BWR makes use of fission detectors incorporated in different detector systems: Start-up, Intermediate and Local Power Range (Morgan, 1970). The Local Power Range Monitoring (LPRM) subsystem is the most elaborate and complex subsystem of the neutron monitoring system. It is composed of numerous in-core fission chambers, typically placed in four axial planes of the reactor core, arranged along an in-core assembly. To provide core wide coverage, a geometrical array of the in-core assemblies is used. A typical array is shown in Fig. 1.

The neutronic power signals obtained from the Local Power Range Monitors are difficult to analyse. Several techniques exist to detect and classify the possible oscillations in a BWR, as the one presented in (Van der Hagen et al., 1994), where the out-of-phase oscillations contributions are separated subtracting the contributions of signals from LPRMs placed in an opposite direction with respect to the symmetry line of the core. The main disadvantage of this method is that the determination of the symmetry line of the core is not an easy task, and to obtain accurate results, a large number of instrumented LPRMs are needed. Other possibility is to use a modal decomposition of the signals from the LPRMs in the core (Verdú et al., 1998) using the dominant Lambda modes previously computed for a given configuration of the reactor core (Verdú et al., 1994). To use this technique it is necessary to 
compute previously the dominant modes of a static configuration of the reactor core. This computation is expensive from the computational point of view and requires a set of nuclear cross-sections for the core configuration. In this way, other options have been investigated that avoid this computation. The Principal Component Analysis (PCA), considers the signals provided by all instrumented LPRMs constructing an information matrix, and studying the characteristics of the spectrum of the dominant singular values of this matrix (Ginestar et al., 2006). Another option is the Independent Component Analysis (ICA) that computes experimental modes for the LPRMs signals using a statistical independence criterion (Ginestar et al., 2011).

Since there is a physical interpretation for the amplitudes evolution of the different neutronic harmonics, especially in the analysis of instability events, a combination of these tools becomes useful to analyse this kind of events, distinguishing the contributions of the in-phase oscillations and the out-of-phase oscillations. Apart from the Lambda modes other kind of modes can be defined for the neutron diffusion equation, such as the Alpha modes (time-eigenvalues problem). The Alpha modes problem is basic in the field of nuclear reactor physics (Bell and Glasstone, 1970), and it is important for subcriticality continuous monitoring techniques (see, for example, (Uhrig, 1970; Williams, 1974)). Recently, it has been proposed in Ref. Kobayashi (2005) to use the quasi-static method to solve time dependent source problems using as weight function the adjoint Alpha fundamental eigenmode, which is shown to be better than the adjoint Lambda eigenmode, especially for the analysis of subcritical systems. This is justified because the use of the adjoint Alpha modes eliminates the first order error in the change of the flux introduced by the use of the adjoint Lambda mode as weighting function.

The Alpha modes can be efficiently computed for a commercial reactor (Verdú et al., 2010), in this way, to complete the set of tools available to analyse the LPRM signals provided during an instability event, in this paper, different power modal decomposition methods using the Alpha modes are proposed and compared with the ones performed using the Lambda modes.

The rest of the paper is structured as follows: sections 2 and 3 are devoted, respectively, to review the Lambda modes, the Alpha modes and their properties. The different modal decompositions proposed using the Lambda modes and the Alpha modes are presented in section 4. The results of the different modal decompositions performed for some of the LPRMs signals provided in the Ringhals stability benchmark are shown in section 5. Finally, the main conclusions of the paper are summarized in section 6 . 


\section{Lambda modes}

To obtain the Lambda modes equation (Henry, 1982), our starting point is the two-energy groups approximation of the neutron diffusion equation,

$$
\begin{aligned}
& \left(v^{-1}\right) \frac{\partial \phi}{\partial t}+\mathcal{L} \phi=(1-\beta) \mathcal{M} \phi+\sum_{k=1}^{K} \lambda_{k}^{d} \mathcal{C}_{k} \chi \\
& \frac{d \mathcal{C}_{k}}{d t}=\beta_{k} \mathcal{M}_{1} \phi-\lambda_{k}^{d} \mathcal{C}_{k}, \quad k=1, \ldots, K
\end{aligned}
$$

where

$$
\mathcal{L}=\left(\begin{array}{cc}
-\vec{\nabla}\left(D_{1} \vec{\nabla}\right)+\Sigma_{a 1}+\Sigma_{12} & 0 \\
-\Sigma_{12} & -\vec{\nabla}\left(D_{2} \vec{\nabla}\right)+\Sigma_{a 2}
\end{array}\right), \quad\left(v^{-1}\right)=\left(\begin{array}{cc}
\frac{1}{v_{1}} & 0 \\
0 & \frac{1}{v_{2}}
\end{array}\right)
$$

and

$$
\mathcal{M}=\left(\begin{array}{cc}
\nu \Sigma_{f 1} & \nu \Sigma_{f 2} \\
0 & 0
\end{array}\right), \quad \mathcal{M}_{1}=\left(\nu \Sigma_{f 1} \nu \Sigma_{f 2}\right), \quad \phi=\left(\begin{array}{l}
\phi_{1} \\
\phi_{2}
\end{array}\right), \quad \chi=\left(\begin{array}{l}
1 \\
0
\end{array}\right) .
$$

Criticality can be forced by dividing the fission nuclear cross sections by a positive number, $\lambda$, obtaining the steady state equations

$$
\begin{aligned}
& \mathcal{L} \phi=(1-\beta) \frac{\mathcal{M}}{\lambda} \phi+\sum_{k=1}^{K} \lambda_{k}^{d} \mathcal{C}_{k} \chi \\
& 0=\beta_{k} \frac{\mathcal{M}}{\lambda} \phi-\lambda_{k}^{d} \mathcal{C}_{k} \chi
\end{aligned}
$$

that is,

$$
\mathcal{L} \phi=(1-\beta) \frac{\mathcal{M}}{\lambda} \phi+\sum_{k=1}^{K} \beta_{k} \frac{\mathcal{M}}{\lambda} \phi .
$$

Taking into account that $\sum_{k=1}^{K} \beta_{k}=\beta$, we obtain the Lambda modes equation

$$
\mathcal{L} \phi_{n}=\frac{1}{\lambda_{n}} \mathcal{M} \phi_{n}
$$

where $\lambda_{n}$ are the Lambda eigenvalues and $\phi_{n}$ their corresponding Lambda modes. A static configuration of the reactor is described by the dominant Lambda eigenvalue, which is the k-effective, and its corresponding eigenmode describes the stationary neutron flux distribution in the reactor core. 
The adjoint problem associated with (4) is given by

$$
\mathcal{L}^{\dagger} \phi_{n}^{\dagger}=\frac{1}{\lambda_{n}} \mathcal{M}^{\dagger} \phi_{n}^{\dagger}
$$

where

$$
\mathcal{L}^{\dagger}=\left(\begin{array}{cc}
-\vec{\nabla}\left(D_{1} \vec{\nabla}\right)+\Sigma_{a 1}+\Sigma_{12} & -\Sigma_{12} \\
0 & -\vec{\nabla}\left(D_{2} \vec{\nabla}\right)+\Sigma_{a 2}
\end{array}\right), \quad \mathcal{M}^{\dagger}=\left(\begin{array}{c}
\nu \Sigma_{f 1} \\
\nu \Sigma_{f 2}
\end{array}\right) .
$$

The Lambda modes, $\phi_{n}$, and their adjoint Lambda modes, $\phi_{m}^{\dagger}$, satisfy a biorthogonality relation of the form

$$
\left\langle\phi_{m}^{\dagger}, \mathcal{M} \phi_{n}\right\rangle=\int_{R} d \vec{r} \phi_{m}^{\dagger} \mathcal{M} \phi_{n}=\delta_{m, n}\left\langle\phi_{m}^{\dagger}, \mathcal{M} \phi_{n}\right\rangle
$$

where $R$ is the volume defined by the reactor core and $\delta_{m, n}$ is Kronecker's delta.

\section{Alpha modes}

To obtain the Alpha modes equation (Bell and Glasstone, 1970), the starting point is again the neutron diffusion equation,

$$
\left(v^{-1}\right) \frac{\partial \phi}{\partial t}+\mathcal{L} \phi=(1-\beta) \mathcal{M} \phi+\sum_{k=1}^{K} \lambda_{k}^{d} \mathcal{C}_{k} \chi
$$

and the delayed neutron precursors are assumed to be in steady state

$$
0=\beta_{k} \mathcal{M}_{1} \phi-\lambda_{k}^{d} \mathcal{C}_{k}
$$

This implies

$$
\left(v^{-1}\right) \frac{\partial \phi}{\partial t}+\mathcal{L} \phi=(1-\beta) \mathcal{M} \phi+\sum_{k=1}^{K} \beta_{k} \mathcal{M} \phi
$$

that is,

$$
\left(v^{-1}\right) \frac{\partial \phi}{\partial t}+\mathcal{L} \phi=\mathcal{M} \phi
$$

Assuming the time factorization

$$
\phi(\vec{r}, t)=e^{\alpha t} \psi(\vec{r})
$$


for the neutronic flux, we obtain the Alpha modes equation (Modak and Gupta, 2007), (Verdú et al., 2010),

$$
(-(v) \mathcal{L}+(v) \mathcal{M}) \psi_{n}=\alpha_{n} \psi_{n},
$$

where $\alpha_{n}$ are the Alpha eigenvalues and $\psi_{n}$ their corresponding Alpha modes. It is worth to be remarked that a critical configuration of the reactor corresponds to the value $\alpha=0$ and, while for the Lambda modes problem we are interested on computing the dominant eigenmodes (the ones with the associated eigenvalue with largest magnitude), for the Alpha modes problem we will be interested on computing the Alpha modes whose corresponding eigenvalues have the smallest magnitude.

The adjoint Alpha modes problem is given by

$$
\left(-\mathcal{L}^{\dagger}(v)+\mathcal{M}^{\dagger}(v)\right) \psi_{n}^{\dagger}=\alpha_{n} \psi_{n}^{\dagger}
$$

If the Alpha modes are non degenerate, the Alpha modes and their corresponding adjoint modes are biorthogonal, that is

$$
\left\langle\psi_{m}^{\dagger}, \psi_{n}\right\rangle=\int_{R} d \vec{r} \psi_{m}^{\dagger} \psi_{n}=\delta_{m, n}\left\langle\psi_{m}^{\dagger}, \psi_{n}\right\rangle
$$

Using the Alpha modes equation (8) and the adjoint Lambda modes, we can write

$$
\left\langle\phi_{n}^{\dagger},(-\mathcal{L}+\mathcal{M}) \psi_{n}\right\rangle=\alpha_{n}\left\langle\phi_{n}^{\dagger},\left(v^{-1}\right) \psi_{n}\right\rangle,
$$

thus,

$$
-\left\langle\mathcal{L}^{\dagger} \phi_{n}^{\dagger}, \psi_{n}\right\rangle+\left\langle\phi_{n}^{\dagger}, \mathcal{M} \psi_{n}\right\rangle=\alpha_{n}\left\langle\phi_{n}^{\dagger},(v)^{-1} \psi_{n}\right\rangle,
$$

and using the adjoint Lambda modes equation (5), we obtain a relation between the Lambda modes and the Alpha modes given by

$$
\alpha_{n}=\frac{\left(1-\frac{1}{\lambda_{n}}\right)}{\frac{\left\langle\phi_{n}^{\dagger},\left[v^{-1}\right] \psi_{n}\right\rangle}{\left\langle\phi_{n}^{\dagger}, \mathcal{M} \psi_{n}\right\rangle}} .
$$

\section{LPRM modal decomposition}

\subsection{Lambda modes}

As it has been exposed above, BWR reactors have different number of Local Power Range Monitors installed in its core, which measure the neutronic power in different positions of the core at different levels. 
In the two energy groups approximation, the neutronic flux is composed of the fast and thermal fluxes

$$
\phi=\left(\begin{array}{l}
\phi_{1} \\
\phi_{2}
\end{array}\right),
$$

and the local neutronic power distribution is of the form (Verdú et al., 1998)

$$
P=K\left(\Sigma_{f 1} \phi_{1}+\Sigma_{f 2} \phi_{2}\right),
$$

where $K$ is a normalization constant.

Assuming that the neutronic flux can be described as a linear combination of $N_{m}$ Lambda modes

$$
\left(\begin{array}{l}
\phi_{1} \\
\phi_{2}
\end{array}\right)=\sum_{n=1}^{N_{m}} a_{n}\left(\begin{array}{c}
\phi_{1, n} \\
\phi_{2, n}
\end{array}\right),
$$

the neutronic power can be written as

$$
P=\sum_{n=1}^{N_{m}} a_{n}\left(\Sigma_{f 1} \phi_{1, n}+\Sigma_{f 2} \phi_{2, n}\right)=\sum_{n=1}^{N_{m}} a_{n} P_{n},
$$

where $P_{n}$ are the power modes

$$
P_{n}=\Sigma_{f 1} \phi_{1, n}+\Sigma_{f 2} \phi_{2, n}
$$

Assuming that the average number of neutrons per fission, $\nu$, is the same for the fast and thermal groups and that it remains constant for the whole reactor core, by using the biorthogonality relation (6), we obtain the amplitudes, $a_{n}$, of the power modal decomposition as,

$$
a_{n}=\frac{\nu}{\left\langle\phi_{n}^{\dagger}, \mathcal{M} \phi_{n}\right\rangle} \int_{R} d \vec{r} \phi_{1, n}^{\dagger} P
$$

A discrete version of expression (13) works well if the power distribution is known in all the nodes of the core discretization. Unfortunately, in a real situation the only information we have is the neutronic power measured by the LPRMs.

To simplify the process, we consider that the LPRM signal contributions correspond to an average plane of the reactor. These signals are

$$
L_{1}(r T), L_{2}(r T), \ldots, L_{p}(r T), \quad r=1,2, \ldots, N_{t},
$$

and $T$ is the sampling time of the acquisition system. Each signal $L_{l}(r T)$ is considered to be placed in the spatial coordinates $\left(x_{l}, y_{l}\right)$. Also it is assumed 
that the signals have zero mean. Using the power modal decomposition (12), it can be assumed that the LPRM signals can be expanded as

$$
L_{l}(r T)=\sum_{n=1}^{N_{m}} a_{n}(r T) P_{n}\left(x_{l}, y_{l}\right)
$$

To estimate the amplitudes $a_{n}(r T)$, we follow two ways. First, analogously to the continuous case, we use the fast adjoint Lambda modes to construct weighting factors $W_{l}\left(x_{l}, y_{l}\right)=\phi_{1, l}^{\dagger}\left(x_{l}, y_{l}\right)$. These factors are not orthogonal to the power modes if only the positions of the LPRMs are considered, but they can be used to construct a system of linear equations of the form

$$
\sum_{l=1}^{p} \phi_{1, m}^{\dagger}\left(x_{l}, y_{l}\right) L_{l}(r T)=\sum_{n=1}^{N_{m}}\left(\sum_{l=1}^{p} \phi_{1, m}^{\dagger}\left(x_{l}, y_{l}\right) P_{n}\left(x_{l}, y_{l}\right)\right) a_{n}(r T)
$$

with $m=1, \ldots, N_{m}$. Solving these systems for $r=1, \ldots, N_{t}$, the time evolution of the amplitudes of the different power modes are obtained.

A second procedure used to compute the power mode amplitudes is based on a least squares criterion. Using this criterion it is assumed that the amplitudes, $a_{n}(r T)$, make minimum the square error

$$
\varepsilon^{2}=\sum_{l=1}^{p}\left(L_{l}(r T)-\sum_{n=1}^{N_{m}} a_{n}(r T) P_{n}\left(x_{l}, y_{l}\right)\right)^{2} .
$$

Computing the derivative of this error with respect to the amplitudes, we have

$$
\frac{\partial \varepsilon^{2}}{\partial a_{m}(r T)}=0=\sum_{l=1}^{p}\left(L_{l}(r T)-\sum_{n=1}^{N_{m}} a_{n}(r T) P_{n}\left(x_{l}, y_{l}\right)\right) P_{m}\left(x_{l}, y_{l}\right)
$$

obtaining the system of equations

$$
\sum_{l=1}^{p} P_{m}\left(x_{l}, y_{l}\right) L_{l}(r T)=\sum_{n=1}^{N_{m}}\left(\sum_{l=1}^{p} P_{m}\left(x_{l}, y_{l}\right) P_{n}\left(x_{l}, y_{l}\right)\right) a_{n}(r T)
$$

whose solution provides the evolution of the power modes amplitudes using a least squares criterion. System (16) is similar to the system (15) obtained above. The only change is that the power modes are used as weighting factors instead of the fast adjoint Lambda modes. 


\subsection{Alpha modes}

Now, instead of using the Lambda modes as basis functions, it is assumed that the neutronic flux can be expanded in terms of the Alpha modes

$$
\left(\begin{array}{c}
\phi_{1} \\
\phi_{2}
\end{array}\right)=\sum_{n=1}^{N_{m}} c_{n}\left(\begin{array}{c}
\psi_{1, n} \\
\psi_{2, n}
\end{array}\right)
$$

the neutronic power can now be expressed as

$$
P=\sum_{n=1}^{N_{m}} c_{n}\left(\Sigma_{f 1} \psi_{1, n}+\Sigma_{f 2} \psi_{2, n}\right)=\sum_{n=1}^{N_{m}} c_{n} P_{a n},
$$

where $P_{a n}$ are the Alpha power modes,

$$
P_{a n}=\Sigma_{f 1} \psi_{1, n}+\Sigma_{f 2} \psi_{2, n} .
$$

For the Alpha power modes there is not a biorthogonality relation that provides a closed expression for the amplitudes, similar to (13). Thus, a least squares criterion will be used to compute the evolution amplitudes of the Alpha power modes, by solving the linear systems

$$
\sum_{l=1}^{p} P_{a m}\left(x_{l}, y_{l}\right) L_{l}(r T)=\sum_{n=1}^{N_{m}}\left(\sum_{l=1}^{p} P_{a m}\left(x_{l}, y_{l}\right) P_{a n}\left(x_{l}, y_{l}\right)\right) c_{n}(r T) .
$$

\section{Signal analysis results}

To test and compare the performance of the methodologies exposed above, two cases of the Ringhals 1 Stability Benchmark have been considered.

Ringhals 1 is an ABB design BWR with a $2270 \mathrm{MW}$ of nominal thermal power and $11550 \mathrm{~kg} / \mathrm{s}$ of total core rated mass flow. The first analysed case is known as Record 10 of the benchmark. During this event, the reactor was working at $77.7 \%$ of its nominal power and the core flow was of $4104 \mathrm{~kg} / \mathrm{s}(58.2 \%)$. This event has been classified as an in-phase oscillation (Lefvert, 1996). The second case is known as Record 9 of the benchmark and corresponds to LPRMs' measurements when the reactor was working at $72.6 \%$ of its nominal power and a core flow of $3694 \mathrm{~kg} / \mathrm{s}(52.4 \%)$. This event has been classified as an outof-phase oscillation (Lefvert, 1996). Table 1 presents a summary of the core working conditions and the calculated stability characteristics of the global oscillation for both cases. 
Table 1

Stability characteristics of Ringhals reactor for Records 10 and 9.

Power (\%) Flow (\%) Frequency $(\mathrm{Hz}) \quad$ Decay Ratio

(Global oscillation)

\begin{tabular}{ccccc}
\hline Rec. 10 & 77.7 & 58.2 & 0.50 & 0.71 \\
Rec. 9 & 72.6 & 52.4 & 0.56 & 0.80 \\
\hline
\end{tabular}

Table 2

Three dominant Lambda eigenvalues for Records 10 and 9 of Ringhals reactor.

\begin{tabular}{cccc} 
& $\lambda_{1}$ & $\lambda_{2}$ & $\lambda_{3}$ \\
\hline Rec. 10 & 0.99369 & 0.99073 & 0.98716 \\
Rec. 9 & 1.00178 & 0.99493 & 0.99297 \\
\hline
\end{tabular}

Table 3

Three smallest Alpha eigenvalues for Records 10 and 9 of Ringhals reactor.

\begin{tabular}{cccc} 
& $\alpha_{1}$ & $\alpha_{2}$ & $\alpha_{3}$ \\
\hline Rec. 10 & -106.1040 & -281.7486 & -321.9185 \\
Rec. 9 & 41.9364 & -119.4661 & -165.0216 \\
\hline
\end{tabular}

\subsection{Lambda modes}

The first three dominant Lambda modes for Records 10 and 9 have been computed using the LAMBDA code (Verdú et al., 1999, 2005). The computed values for the three dominant eigenvalues for Records 10 and 9 are shown in Table 2.

The shapes of an average plane of the power modes distribution associated with these eigenvalues are presented in Fig. 2 and Fig. 3. For both configurations we obtain, consecutively, the fundamental mode and two azimuthal modes.

\subsection{Alpha modes}

The first three Alpha modes with smallest magnitude have been also computed for Records 10 and 9 (Verdú et al., 2010). The obtained values for the three smallest eigenvalues for Records 10 and 9 are shown in Table 3.

The shapes of an average plane of the Alpha power modes associated with these eigenvalues are presented in Fig. 4 and Fig. 5. The shape of the Alpha modes are in both cases quite similar to ones obtained for the Lambda modes. 

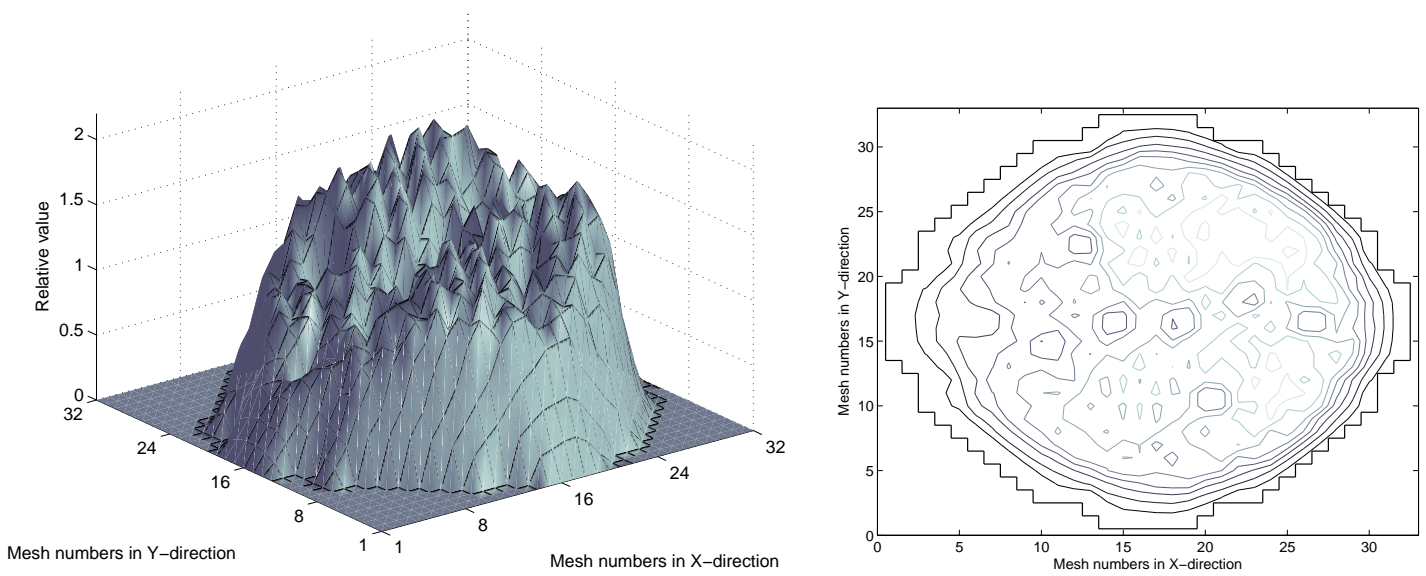

First mode (Fundamental)
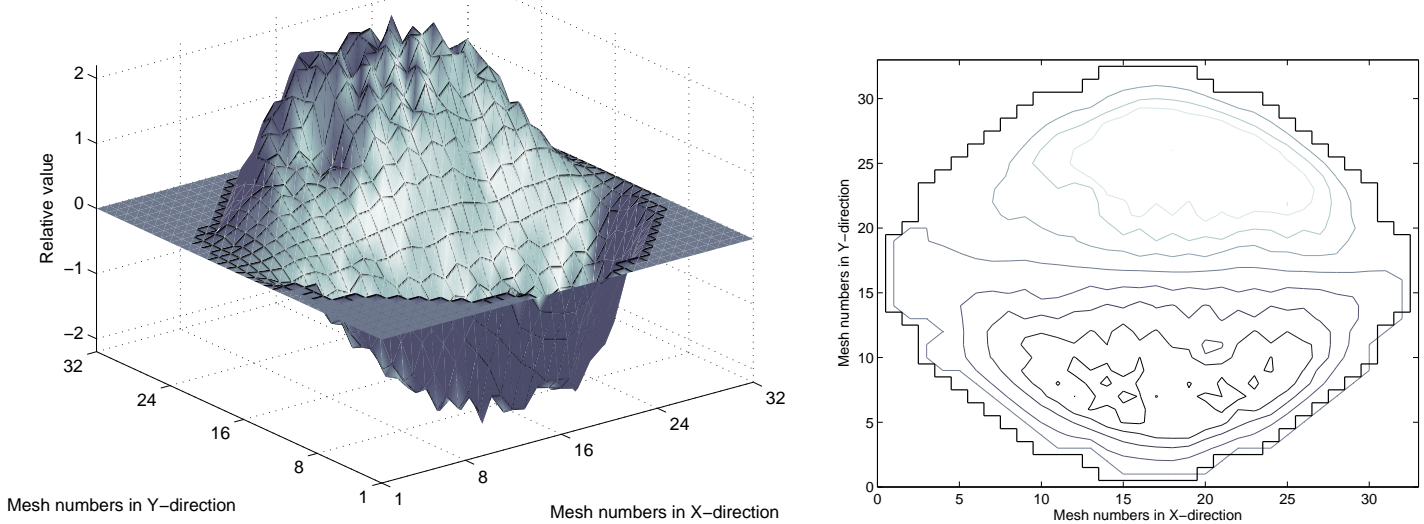

Second mode (Azimuthal)
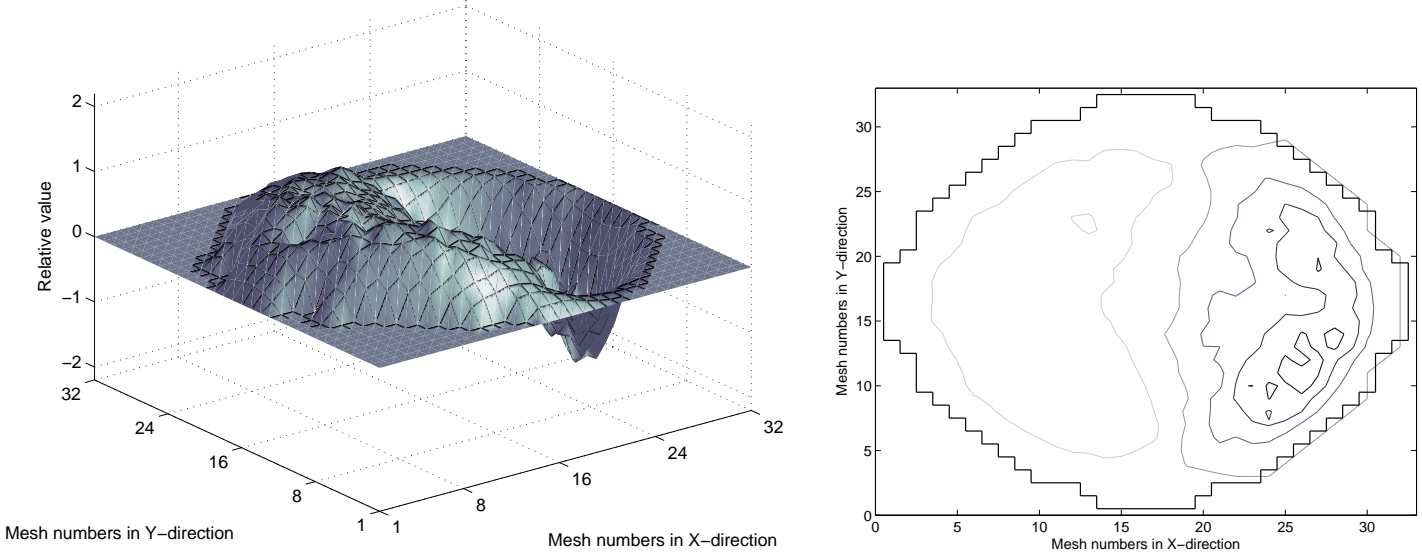

Third mode (Azimuthal)

Fig. 2. First three power modes for the Record 10 configuration of Ringhals reactor. Surface and contour representation of the axial average plane of the modes. 

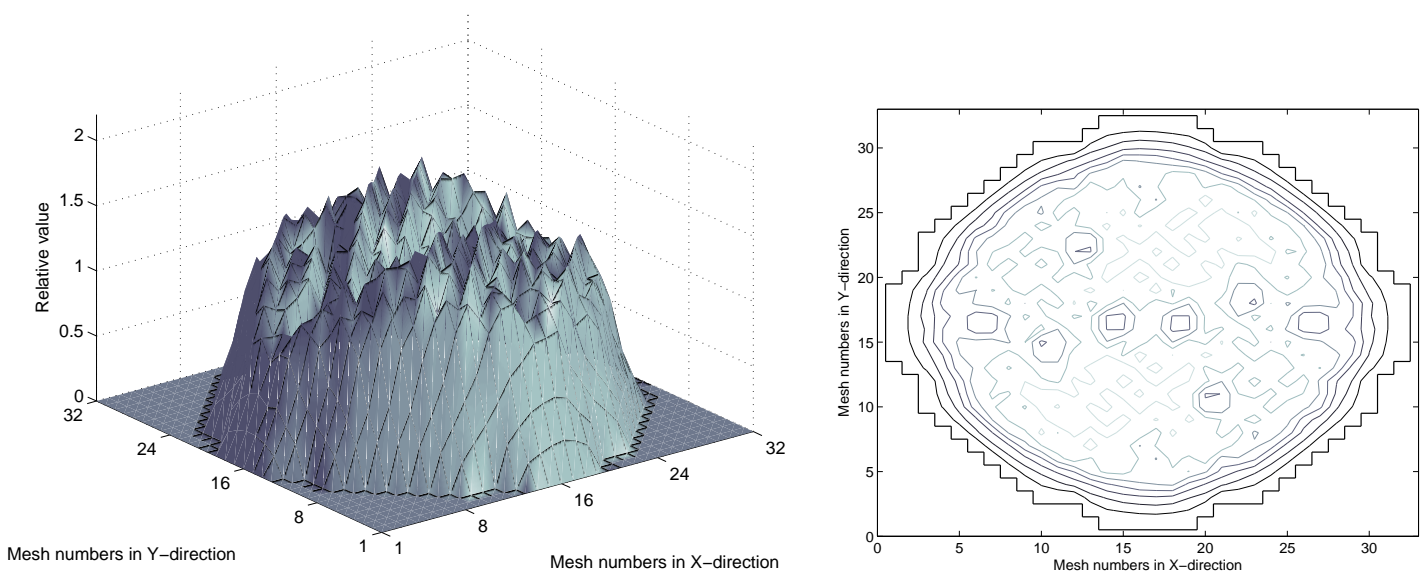

First mode (Fundamental)
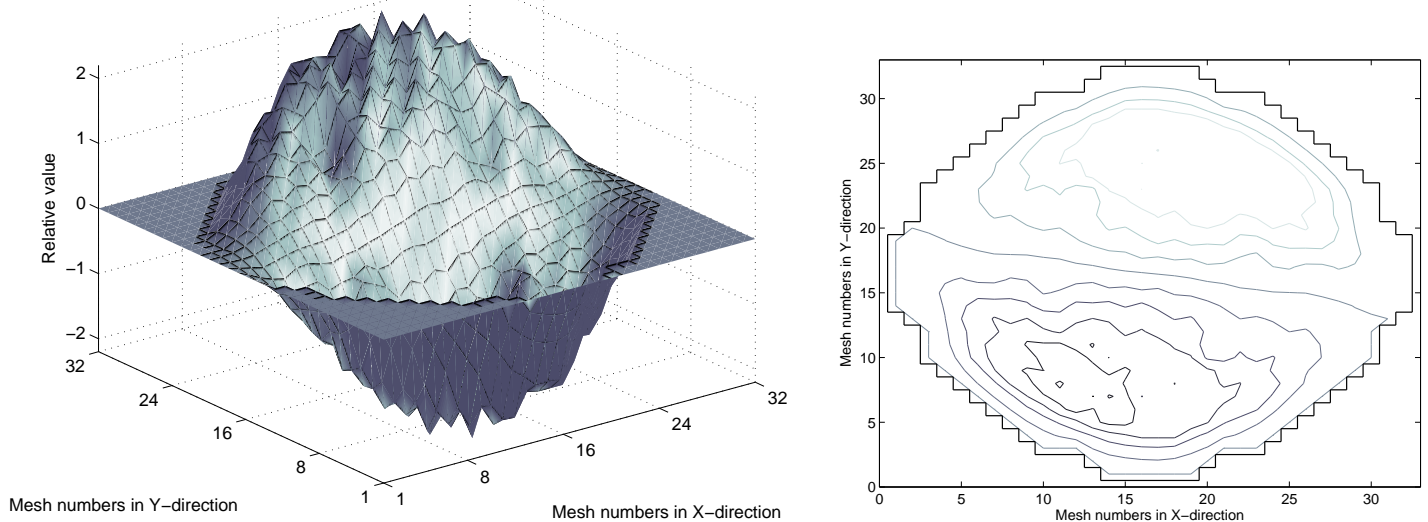

Second mode (Azimuthal)
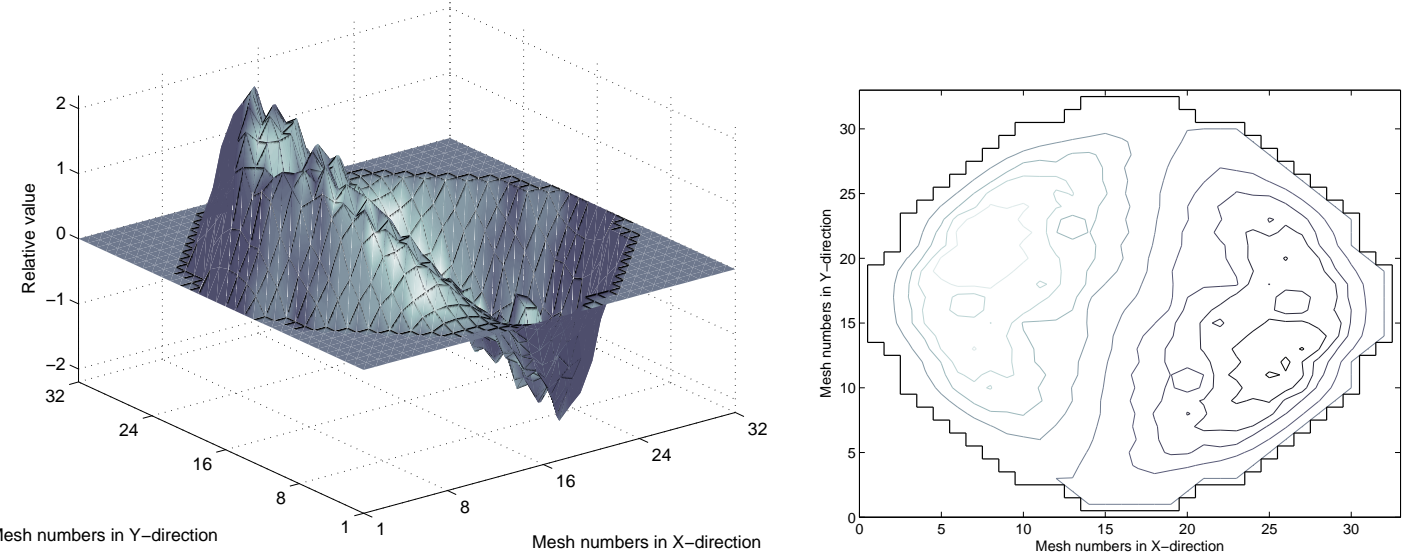

Third mode (Azimuthal)

Fig. 3. First three power Lambda modes for the Record 9 configuration of Ringhals reactor. Surface and contour representation of the axial average plane of the modes. 

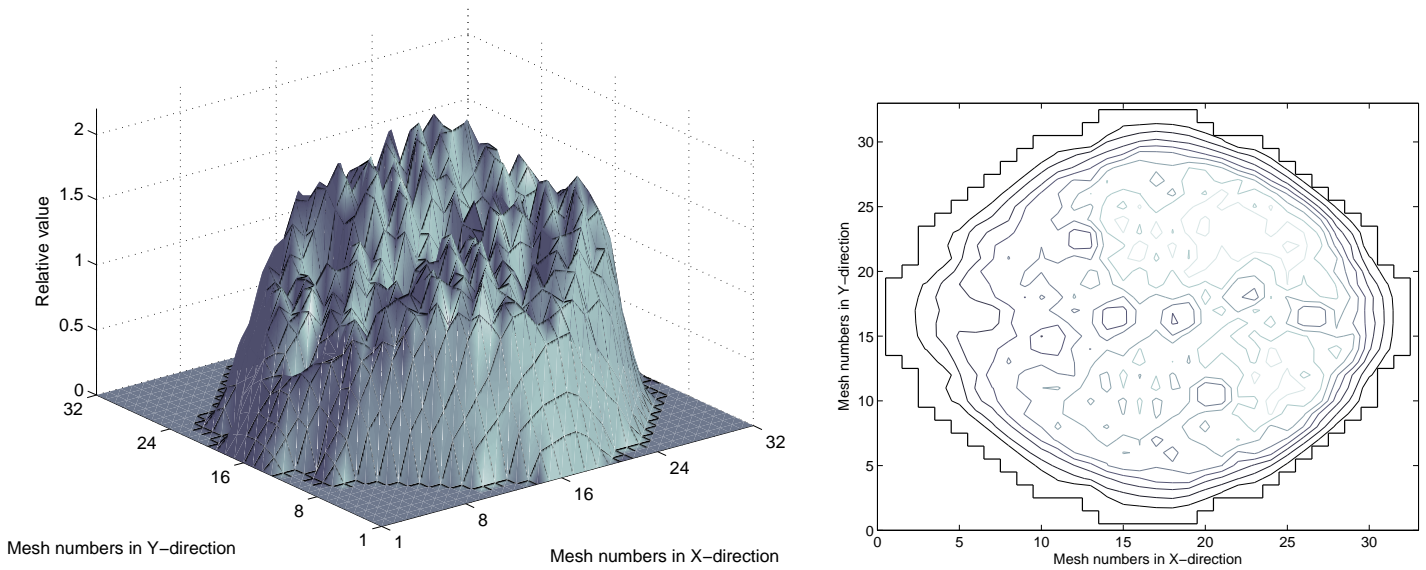

First Alpha power mode (Fundamental)
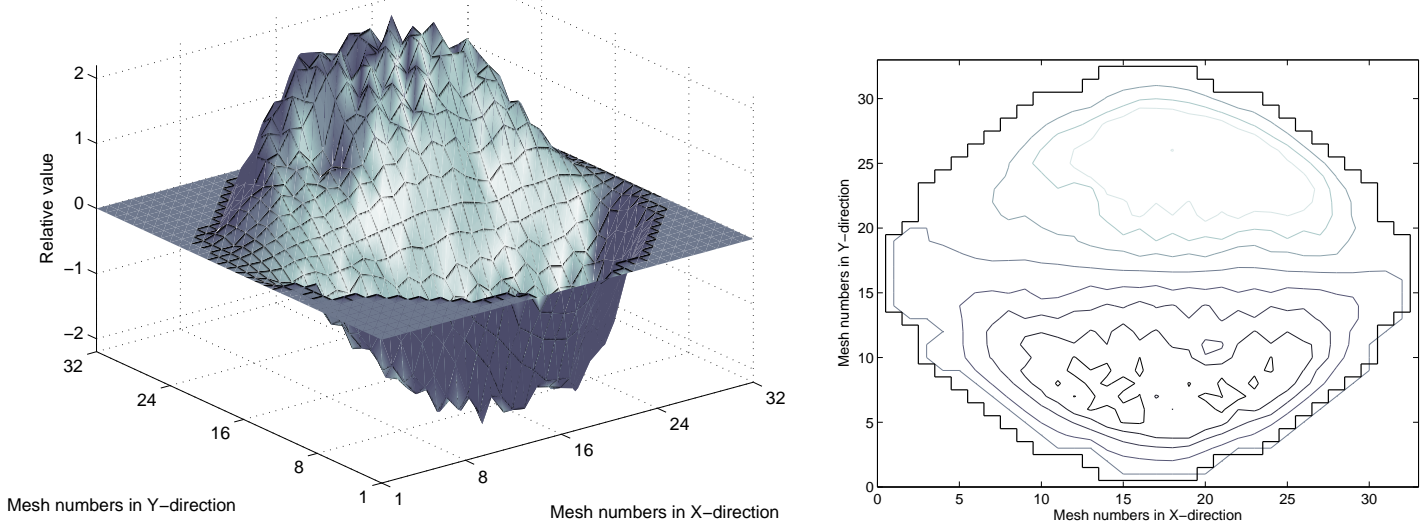

Second Alpha power mode (Azimuthal)
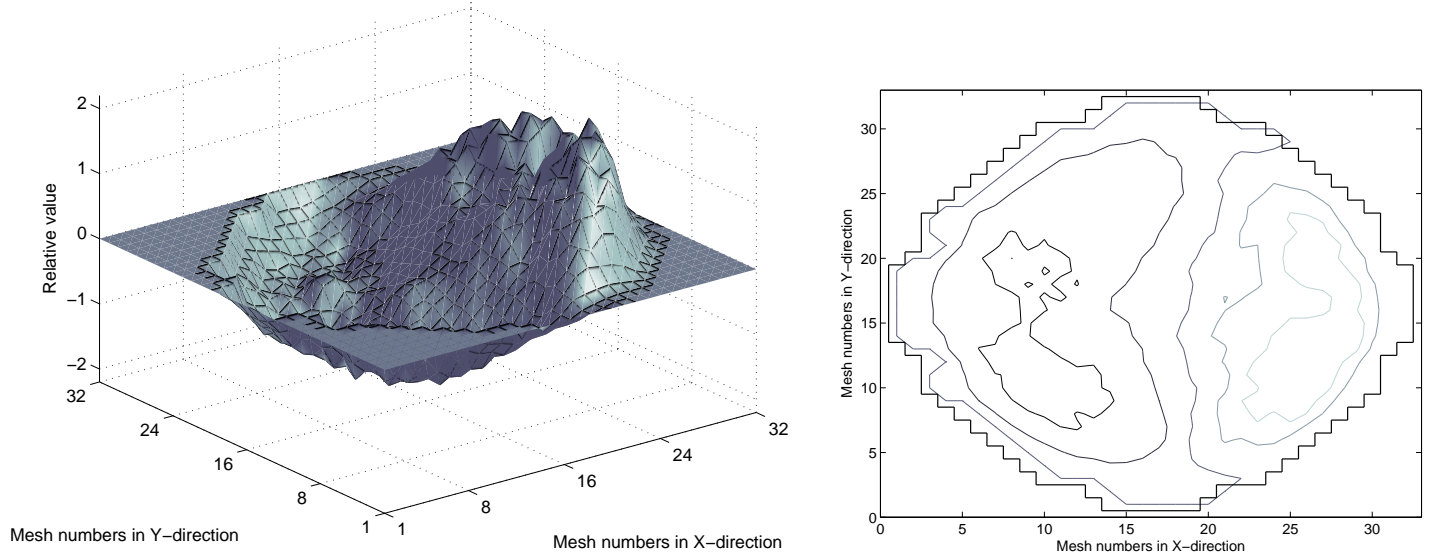

Third Alpha power mode (Azimuthal)

Fig. 4. Smallest three Alpha power modes for the Record 10 configuration of Ringhals reactor. Surface and contour representation of the axial average plane of the modes. 

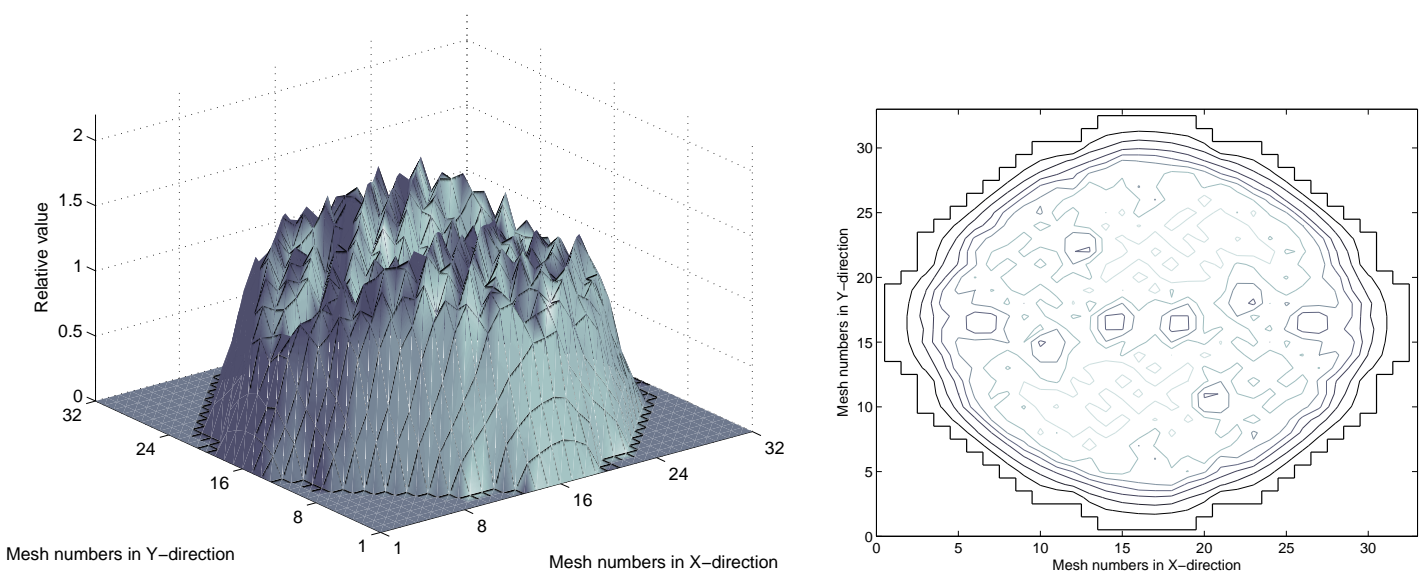

First Alpha power mode (Fundamental)
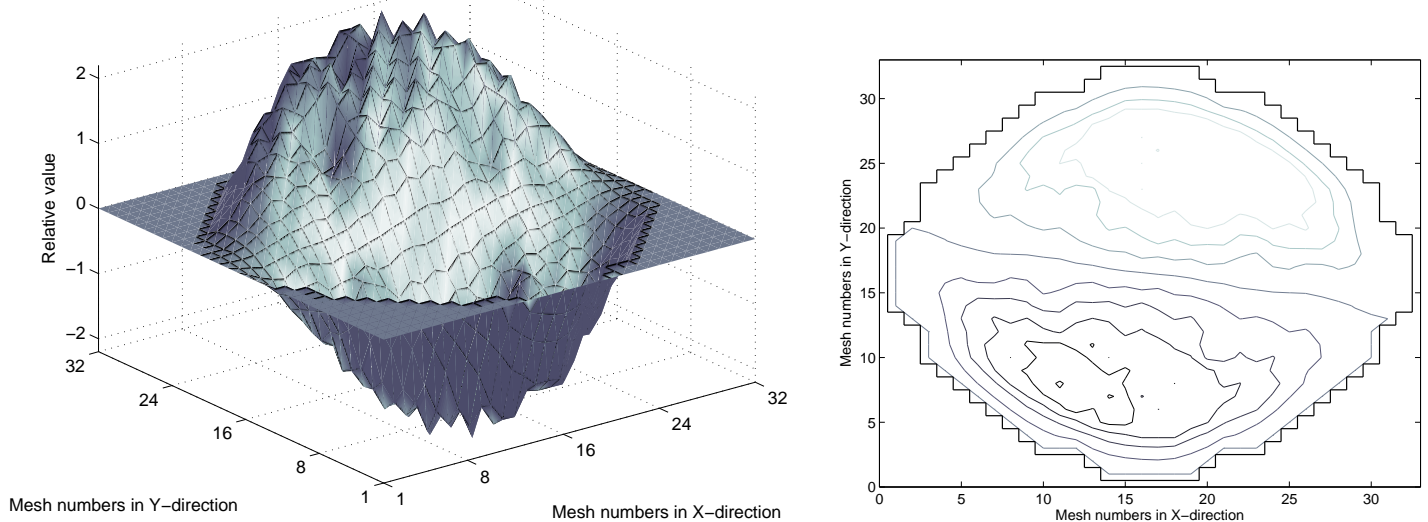

Second Alpha power mode (Azimuthal)
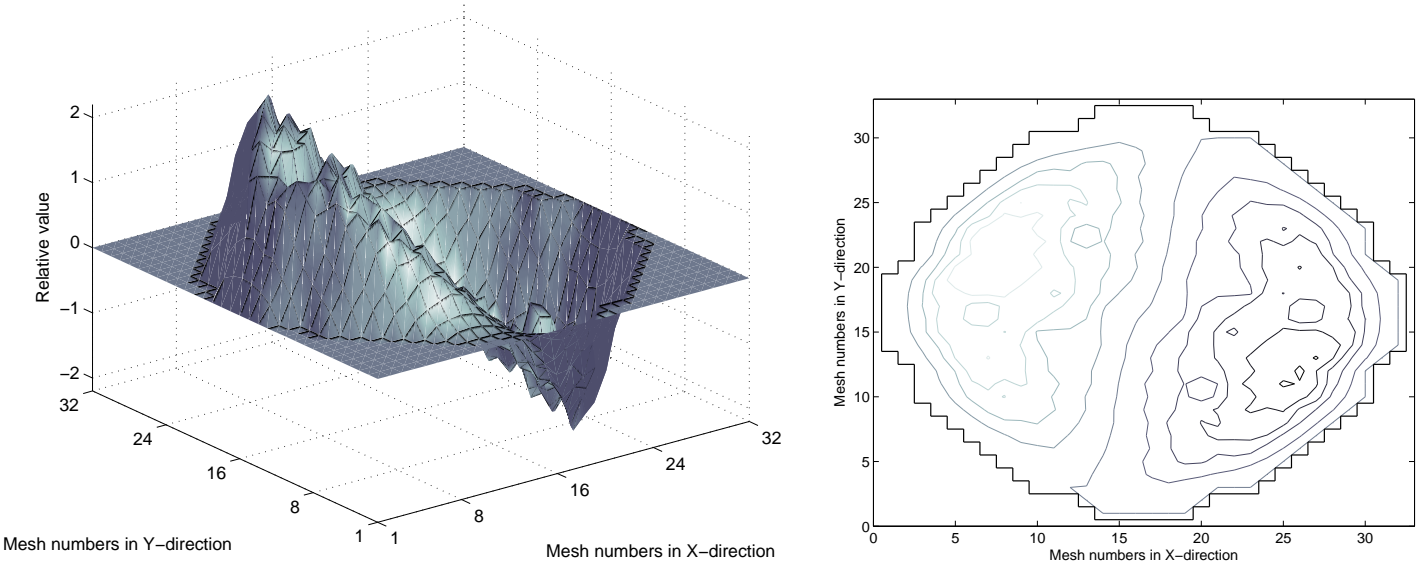

Third Alpha mode (Azimuthal)

Fig. 5. Smallest three Alpha power modes for the Record 9 configuration of Ringhals reactor. Surface and contour representation of the axial average plane of the modes. 


\subsection{Signals decompositions}

For both Records 10 and 9, we have considered signals of the LPRMs placed in two axial planes with 36 detectors per plane. These signals have a sampling time of $T=0.08 \mathrm{~s}$ and we have considered $N_{t}=1000$ samples for each signal. The signals for the two axial planes are averaged and the mean of each signal is subtracted.

The three computed eigenmodes are used to perform the LPRM Modal Decomposition of Records 10 and 9 and in order to compare the power modal decompositions using the Lambda and the Alpha modes, only the least squares criterion for the computation of the amplitudes evolution is used.

The real LPRM number 30 for Record 10 together with its Lambda power modal reconstruction by means of expression (12) and its Alpha power modal reconstruction with expression (17) are shown in Figure 6. LPRM number 30 of Record 9 and its reconstructions are shown in Figure 7. To give a quantitative measure of the error obtained for the signals reconstruction using the Lambda modes we introduce the relative error

$$
\varepsilon_{l}=\left(\frac{\sum_{r=1}^{N_{t}} \sum_{l=1}^{p}\left(L_{l}(r T)-\sum_{n=1}^{N_{m}} a_{n}(r T) P_{n}\left(x_{l}, y_{l}\right)\right)^{2}}{\sum_{r=1}^{N_{t}} \sum_{l=1}^{p}\left(L_{l}(r T)\right)^{2}}\right)^{\frac{1}{2}}
$$

Similarly, for the signals reconstruction using the Alpha modes we introduce the error

$$
\varepsilon_{a}=\left(\frac{\sum_{r=1}^{N_{t}} \sum_{l=1}^{p}\left(L_{l}(r T)-\sum_{n=1}^{N_{m}} c_{n}(r T) P_{a n}\left(x_{l}, y_{l}\right)\right)^{2}}{\sum_{r=1}^{N_{t}} \sum_{l=1}^{p}\left(L_{l}(r T)\right)^{2}}\right)^{\frac{1}{2}}
$$

The relative errors obtained in the reconstruction of the 36 signals of Record 10 and Record 9 using the Lambda modes and the Alpha modes are shown in Table 4.

It is observed that the obtained relative errors are quite similar for both reconstructions, being the reconstruction obtained with the Lambda modes slightly better than the reconstruction obtained using the Alpha modes. Also, it is interesting to remark that similar results are obtained if the adjoint modes are 
Table 4

Relative errors for the reconstruction of the signals of Records 10 and 9 of Ringhals reactor using the Lambda modes and the Alpha modes.

\begin{tabular}{ccc} 
& $\varepsilon_{l}$ & $\varepsilon_{a}$ \\
\hline Rec. 10 & 0.536 & 0.543 \\
Rec. 9 & 0.435 & 0.451 \\
\hline
\end{tabular}

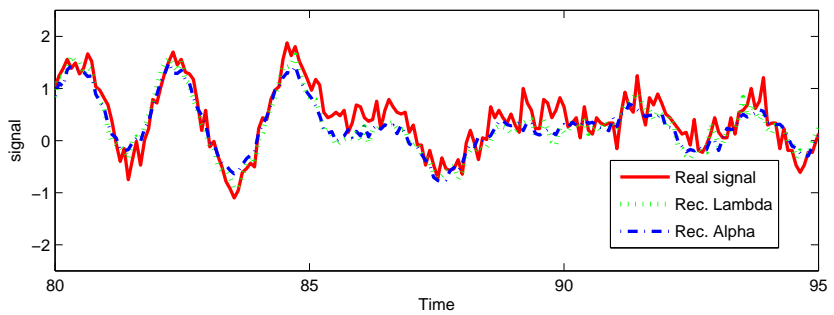

Fig. 6. LPRM number 30 of Record 10 together with its Lambda Power modal reconstruction and its Alpha power modal reconstruction.

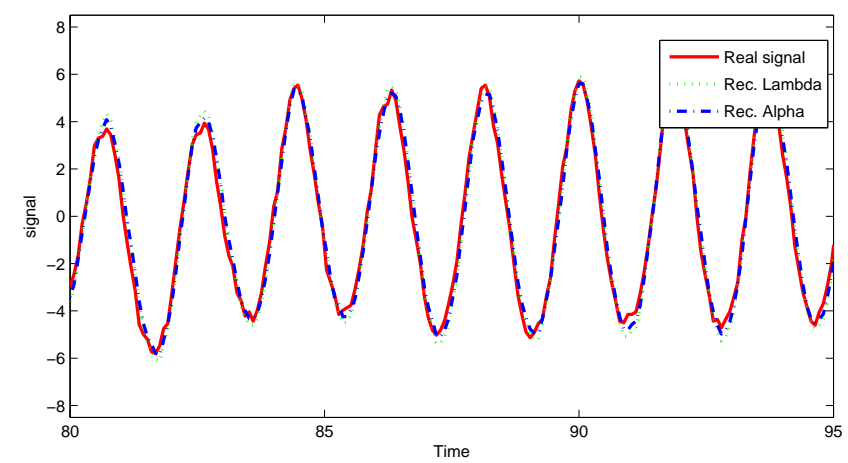

Fig. 7. LPRM number 30 of Record 9 together with its Lambda Power modal reconstruction and its Alpha power modal reconstruction.

used to construct weighting factors to compute the power modes amplitudes evolution.

The results for the time evolution of the amplitudes of the different Lambda power modes corresponding to Record 10 are shown in Fig. 8, and the corresponding to Record 9 in Fig. 9. This is a near stable case, with a decay ratio about 0.7 and a fundamental frequency of $0.54 \mathrm{~Hz}$. Nevertheless, from Fig. 9 we can see a developed oscillation for the second and third modal power contributions.

The power modal decomposition of the LPRMs has been also computed using the Alpha-modes. The results obtained for the time evolution of the amplitudes of the different modes corresponding to Record 10 are shown in Fig. 10, and the corresponding to Record 9 in Fig. 11, and the corresponding ones to the Record 10. These results are very similar to the ones obtained with the Lambda 



Fig. 8. Time evolution of the amplitudes of the power lambda modal decomposition for Record 10.
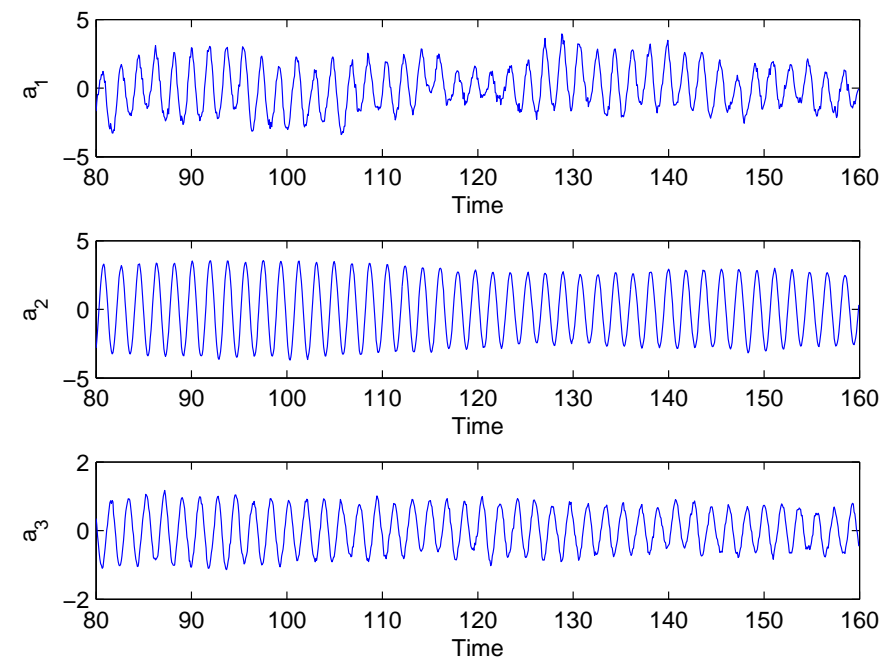

Fig. 9. Time evolution of the amplitudes of the lambda power modal decomposition for Record 9.

modes.

\section{Conclusions}

For safe operation of BWR reactors it is important to have an efficient system to detect and classify the unstable events using the signals provided by the LPRMs installed in the reactor core. For some instability events, such as the 

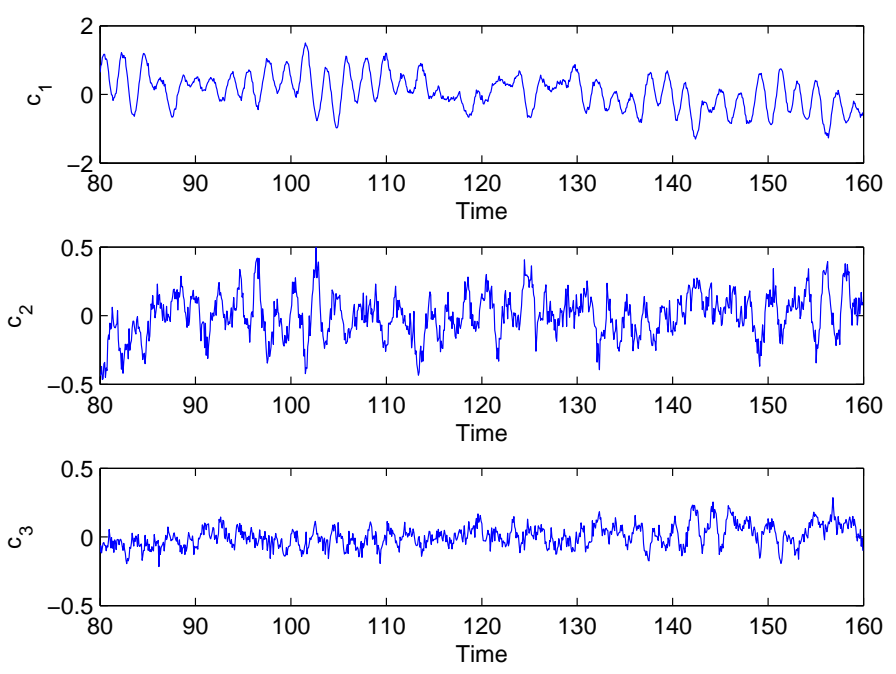

Fig. 10. Time evolution of the amplitudes of the Alpha power modal decomposition for Record 10.
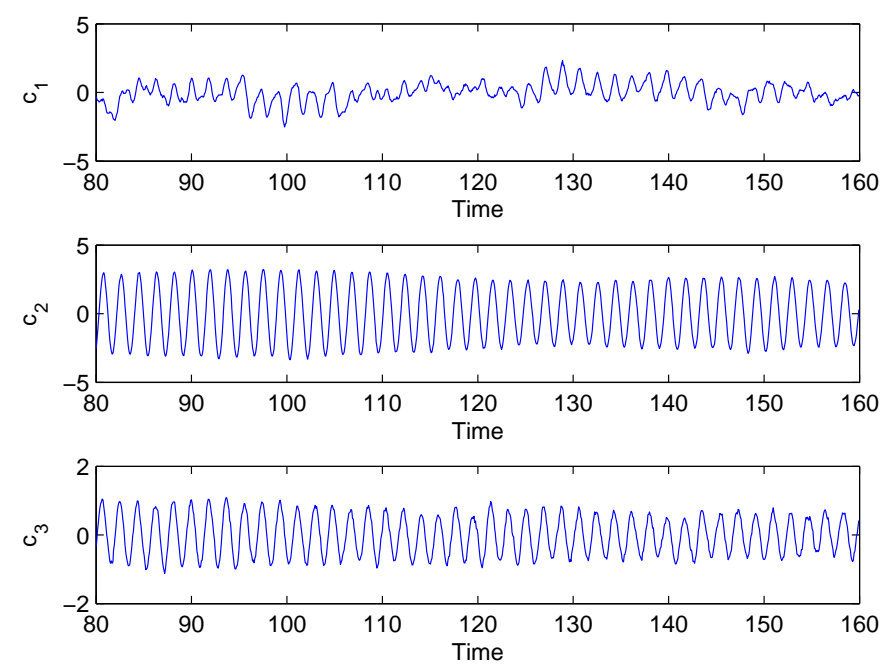

Fig. 11. Time evolution of the amplitudes of the Alpha power modal decomposition for Record 9.

in-phase and the out-of-phase oscillation a modal interpretation has been given associating the in-phase oscillations with the oscillations of the amplitude of the fundamental mode and the out-of-phase oscillations with the oscillations of the azimuthal modes. Several modal equations have been proposed associated with the neutron diffusion equation. In this paper, the Lambda modes and the Alpha modes have been reviewed and a simple method to decompose the LPRMs signals in different power modes contribution using both, the Lambda and the Alpha modes has been proposed and the performance of each kind of modes has been compared studying two cases of Ringhlas 1 stability bench- 
mark. This analysis shows that both the Alpha and the Lambda modes for a nuclear reactor, which is a nearly critical system, have a similar shape and can be successfully used to analyse the different LPRM signals contributions. The Alpha modes and the Lambda modes have different mathematical properties, in this way, they can be used to construct different reduced order models to analyse the BWR stability characteristics.

\section{Acknowledgements}

This work has been partially supported by the Spanish Ministerio de Educación y Ciencia under project ENE2011-22823.

\section{References}

Bell G., S. Glasstone, Nuclear Reactor Theory. Robert E. Krieger Publishing Co., Inc., Malabar, Florida (1970).

Ginestar, D., Verdú, G., Miró, R. Singular system analysis of the local power range monitor (LPRM) readings of a boiling water reactor (BWR) in an unstable event, Int. J. Nuclear Science and Technology 2, 253-256. (2006).

Ginestar, D., Miró, R., Verdú, G., Barrachina, T. Modal processing of the Local Power Range Monitors signals in BWR NPP. Annals of Nuclear Engineering, 38, 11, 2441-2455, (2011).

Henry, A. F., 1982. Nuclear-Reactor Analysis, 4th Edition. The MIT Press.

Kobayashi K. A rigurous weight function for neutron kinetics equations of the quasi-static method for subcritical systems. Annals of Nuclear Energy, 32, 8, 763-776, (2005).

Lefvert, T., 1996. Ringhals I Stability Benchmark NEA/NSC/DOC(96) 22.

March-Leuba, J., Rey, J. M., Coupled thermohydraulic-neutronic instabilities in boiling water nuclear reactors: a review of the state of the art, Nuclear Engineering and Design 145, 97-111, (1993).

Modak R.S., Gupta A., A scheme for the evaluation of dominant timeeigenvalues of a nuclear reactor. Annals of Nuclear Energy, 34, 3, 213-221, (2007).

Morgan, W. R., In-Core Neutron Monitoring System for General Electric Boiling Water Reactors, IEEE Transactions on Nuclear Science NS17 (1), 564571, (1970).

Uhrig, R.E., 1970. Random Noise techniques in Nuclear Reactor Systems. Ronald press. New York.

Van der Hagen, T. H. J. J., Pászit, I., Thomson, O., Melkerson, B., Methods for the determination of the in-phase and out-of-phase stability characteristics of a boiling water reactor, Nuclear Technology 107, 193-214, (1994). 
Verdú, G., Ginestar, D., Vidal, V., Muñoz Cobo, J. L. 3D $\lambda$-modes of the neutron diffusion equation. Annals of Nuclear Energy, 21, 405-421, (1994).

Verdú, G., Ginestar, D., Vidal, V., Miró, R., Modal decomposition method for BWR stability analysis, Journal of Nuclear Science and Technology 35 (8), 538-546, (1998).

Verdú, G., Miró, R., Ginestar, D., Vidal, V., The implicit restarted Arnoldi method and efficient alternative to solve the neutron diffusion equation, Annals of Nuclear Energy 26, 579-593, 1999.

Verdú, G., Ginestar, D., Miró, R., Vidal, V., 2005. Using the Jacobi-Davidson method to obtain the dominant Lambda modes of a nuclear power reactor, Annals of Nuclear Energy 32, 1274-1296.

Verdú, G., Ginestar, D., Román, J., Vidal, V. 3D Alpha Modes of a Nuclear Power Reactor. Journal of Nuclear Science and Technology, 47, 5, 501-514, (2010).

M.M.R. Williams, Random Processes in Nuclear Reactors. Pergamon Press. Oxford. (1974). 